\title{
Index
}

active citizen(s) 120, 195, 208

advanced neoliberal government 2, 222

advocacy groups 11, 59, 91, 144, 145, $153,241,252,253$

Agamben, Giorgio 25, 27, 28

ageing Chapter 11 passim

Amnesty International Ireland 133, 186

anthropological machine 29

see also Agamben, Giorgio

anthropometry 81

anti-tobacco movement 99

ASH Ireland 101, 107

Assessment of Need Chapter 10 passim

asylums 76, 121, 208

audit $10,166,168,169,195$

austerity $4,12,13,138,148,149$, $155,165,166,168,188,242-243$

autism 205, 209, 216, 217, 220, 222

A Vision for Change 16, Chapter 6 passim

bioethics $137,142,149,157$

biological citizenship 141, 143, 155

biopedagogy $73,75,80$

biopower $3,5,6,50,51,74,75,76$, $77,79,80,81,85,89,91$

see also Foucault, Michel

BMI see Body Mass Index

body

the cadaveric 139,144

the civilised 99

collective 107, 108, 141 the dead $16,137,139,14,143$, $149-153,156,250$

the fat 82,83

the healthy $15,72,73,82,84,85$, $86,87,89$

the individual $5,9,13,141,222$

as property 141,142

size $63,66,72,73,82-88$ passim

the social $5,9,13,32,79$

Body Mass Index 6, 15, 47, 48, 50, $51,62,73,83$

Bracken, Pat 122

Bronfenbrenner, Urie 56, 60

calculating selves 10, 205, 217, 219

cancer $10,11,49,73,82,83,90,99$, 101, 103, 104, 109, 138, 154, $162,165,233$

care management 235, 243

Carpenter, Mary 30, 31, 32

Catholic Church 12, 30

Celtic Tiger 13, 212, 239

Chief Bioethics Offer 150-154 passim

Chief Medical Officer 104

childhood 14-15, Chapter 2 passim conceptions of 25-27, 29, 41, 64-66 and Healthy Ireland 37, 38 as 'national asset' $30,35,37$ obesity Chapter 3 passim

Childhood Obesity Surveillance Initiative 54, 64

Children Act (1908) 33, 35

citizens as consumers $2,140,163$, $165,168,170,174$ 
class 9, 30, 32, 167, 185, 191

classification 50, 203, 204, 208, 214, 216, 217, 222

cognitive behavioural therapy 120 , 128

commons 140, 143, 151, 156

community 4, 60, 101, 120, 125, 126, 128, 129, 174, 182, 196, 197, 198, 209, 254

-based services 122, 130, 172, 230, $232,239,241$

development 171

policy 66

rating 185, 190, 197

Community Mental Health Team 129

competition see managed competition; Universal Health Insurance-by Competition

Constitution (Irish) 152, 252

contagion 52, 84, 104, 105, 110

counter-conducts $17,121,131,149$, 156,253

criminal $30,31,32,35$

Critical Voices Network Ireland 122, 123

Cruikshank, Barbara 227, 233

culture 84, 96, 108, 123, 128, 192

Dean, Mitchell

analytics of government 26, 205, 235,250

fields of visibility 16, 121, 124, 184 identity formation 119, 121, 212 regimes of practices $119,121,134$

technologies of citizenship 227, 231, 233

understandings of government 1,4 , 6, 7, 121, 134, 138, 184, 191, 204, 208

delinquency 26, 30, 31, 32, 34

depression 119, 120, 133

diabetes 49, 52, 73, 82, 83, 146, 147, $155,162,241$

diagnosis 51, 82, 129, 213-216, 220, 221, 242

Disability Act 2005 Chapter 10 passim disciplinary power $55,75,76,80,86$ see also Foucault, Michel discipline and bodies 9, 11, 14, 29, 77, 78, 173

discourse

analysis 100, 228

biomedical 73, 117, 122

health promotion 110, 112

public health 98, 99, 110

truth $80,84,85,91$

disease

cardiovascular 73, 82, 150, 162, 165

categories 3, 5, 214

chronic 168

classification 216, 230

conceptualisation 3,73

epidemics 11, 52, 84, 206

indicators $34,50,73,83,84,85$

infectious 105

kidney 146, 155

medical register 31,54

organ 143, 155

risk factors $82,83,84,84$

docile bodies 78, 227, 231, 239

education

and children 29, 33, 34, 132

and obesity 55, 62, 63

and organ donation 149

reformatory 26, 30-32, 40, 41

and sexual health 9

and tobacco control 98

education system

and disability 203, 209, 210, 212, 214, 216

and mental health 119, 124, 135

emigration 148, 165

employability 38, 207

empowerment 162, 195, 227, 234

enterprise society 37

entrepreneur of the self 89, 140, 192

EU see European Union

eugenics 33,81

EU-IMF see European

Union-International Monetary

Fund

Europe 5, 107, 109, 145, 146, 149, $152,168,188,189,198,230$, 233,243 
European Central Bank 148, 149

European Convention on Human

Rights 152

European Union 162, 165, 167, 176

European Union Health

Commissioner 107

European Union-International

Monetary Fund 13

expert knowledge 3, 98, 101, 214,

221,250

\section{FairCare 187}

familism 152, 156, 252

fields of visibility 121, 124, 184

see also Dean, Mitchell

financial management 10, 132, 196, 207

Fine Gael 183, 187, 191, 198, 231

Fine Gael / Labour 137, 182, 185,

188, 239, 240, 241, 242

Fitzgerald, Frances 36, 55, 65

Foucault, Michel

art of government $6,77,97,226$, 227

clinical gaze 49, 73, 86

concepts of power $3,4-8,14,25$, $29,32,36,37,40,72,73$, 75-79, 85, 96, 97, 118, 131, 138-140, 162, 173, 190, 191, 195, 206, 226, 227, 250, 252, 253,255

'conduct of conduct' 1, 6, 16, 78, $85,96,97,138$

game of differentiation 37

game of inequality 26, 36, 37, 40, 41

genealogy 5, 27, 141

lectures on governmentality 6,74 , 77, 119, 206

power/knowledge 4, 8, 50

and subjectification $27,78,80,140$ gender 48, 50, 254

General Medical Service 62

General Practitioners 12, 130, 172

governing at a distance 18, 161, 164, 169, 170, 189, 208

GPs see General Practitioners

Growing Up in Ireland 15, 39, 46, 55, 60,65

\section{GUI see Growing Up in Ireland}

Hacking, Ian 3, 50, 76, 80, 81, 204, 207, 216

Harney, Mary 231

HCCI see Home Care and Community Care Ireland

Health Behaviours in School-Aged Children 39, 40, 168

health inequalities $17,26,35,36,176$, $247,253,254$

Health Information and Quality Authority 12, 18, 211, 214, 234, 239

health insurance $12,13,18,98$, 182-188, 192-194, 196-198

Health Service Executive 12, 13, 59, 122, 127, 129, 132, 148, 204, 209, 211-218 passim, 237-239, 240,242

Healthy Ireland 17, 26, 36, 37, 38, 118, 123, 125, 164, 168, 169, 170, 175, 176, 178, 195

HIQA see Health Information and Quality Authority

Home Care and Community Care Ireland 237

homo economicus 79, 139, 140, 142, 151

HSE see Health Service Executive

identity formation 119, 121, 212 see also Dean, Mitchell

individualisation 161, 255

International Monetary Fund 148, 149

internet 91, 252

Irish Cancer Society 103, 104

Irish Kidney Association 147

Irish Medical Organisation 186

Irish Taskforce on Obesity 54, 55, 56

Joint Committee on Health and

Children 16, 137, 144, 156

kidney transplant 146, 147, 148, 154, 155

life expectancy 76, 165-8 
Lupton, Deborah 5, 6, 8, 9, 49, 63, 64, 98, 99

managed competition 182, 186, 187, 188,191

marketing

digital 86

social 48, 63, 64, 78, 79, 85, 100, 104, 105, 110

techniques $80,85,86$

markets 37, 187, 193, 227

quasi- 192

Martin, Micheál 101-109 passim

McKee, Kim 7, 11, 91, 92, 222, 229, 253

media $6,11,15,46-49$ passim, 55, 59, 63-66, 91, 106, 109, 127, 133, 171, 188, 212, 216, 219, 250

medical

care 182

categories $8,53,87$

discourses 15, 16, 47, 59, 107, 110, $118,129,133,134,150,214$

inspection $26,34,35,40$

institutions 66,101

interventions $6,9,11,12,52,62$, 64, 143, 144, 146, 147, 151, 155

knowledge 8, 26, 30, 52, 76, 119, 122,214

norms $15,25,30,35,76,77,78$, 130, 150, 196, 233

professionals $8,11,15,48,49,59$, 65, 106, 118, 122, 129, 134, $144,146,154$

screening 9

system 155

Medical Card 12, 185

mental health 1, 9, 16, Chapter 6

passim, 168, 178, 209, 219, 220, 252

Mental Health Act (2001) 122, 130

Miller, Peter 4, 7, 10, 13, 18, 37, 79, 98, 183, 189, 192-196, 205-208, 212, 219

see also Rose, Nicholas

mixed economy of healthcare 12, 163 modernity 25, 27 moral

decline 26

health 31

hospital 31

management 32

morality and children 14, 34

motherhood 9, 105

mothers 15, 48, 52, 60, 66, 104, 105, 111,254

National Children's Strategy 38-39

National Disability Authority

Chapter 10 passim

NDA see National Disability Authority neoliberalism 6, 36-37, 74-75, 79, 162-163

see also advanced liberal government neuroscience 130, 132, 133

New Right 6

see also neoliberalism

NHSS see Nursing Home Support Scheme

normalisation 34-35, 65-66, 81 nudge-based policy 86, 89, 173, 177 nursing homes 236-237, 240

Nursing Home Support Scheme 232, 235, 236, 237, 240, 241

obesity $1,6,9,15,146,162,173$, 250, 252-253, 254

and children Chapter 3 passim

'epidemic' 15, 47, 48, 51, 58, 59, 66,173

prevalence of $51,53,54,56-58$

and conflation with overweight 49 , 51-52, 61

measurement of 48-49, 50-51, 81-83

see also Body Mass Index schools 55, 62-63

see also overweight

OECD see Organisation for Economic Co-operation and Development

older people 18, 50, Chapter 11 passim

O’Malley, Pat 3, 10, 99, 161, 204, 248-255 passim

O’Malley, Tim 124

Operation Transformation 51, 66 
organ

donation 50, 251

retention scandal 138

scarcity 124

transplantation 137, 152, 157

Organisation for Economic

Co-operation and

Development 53, 168, 230

overweight Chapter 4 passim, 46

conflation with obesity 49,51

measurement of $51,72,77,81,83$

prevalence amongst Irish children 53, 54, 55, 56, 57 see also obesity

parenting 35,48

parents $15,30,35,46,60,62-66$

passim, 138, 208, 210, 212, 214,

215, 216-217, 220, 250, 254

see also mothers

partnership 36, 56, 133, 149, 170,

171, 177, 209, 211

pastoral power 90

see also Foucault, Michel

paternalism 15, 162

patient(s)

categories of 13, 185, 194

as citizens 1, 137, 148, 172, 185

as consumers $2,163,165,166$, 191, 192, 193, 195, 196, 199, 238

as experts 162

and mental health 130,131

moral 31

and obesity 62,83

and organ transplantation 146-149, $154,155,157$

rational 98

satisfaction surveys 98,166

patients' organisations 11, 143, 191

see also advocacy groups

people with disabilities 10, Chapter 10

passim

performance indicators 10, 166, 241

Phillips, Anne 139, 141, 151, 156

population(s)

ageing 230, 242

and biopower 55, 76-77, 80, 85, 222 as governable entities 204, 206, 207, 212

health of $5,6,14,81,83,92,161$, $166,169,171,175$

management of 1, 3, 31, 204, 227, 229

and mental health 124, 125, 126, 134

and obesity 88,89

childhood 51

and organ donation 146, 148

and private health insurance 12-13, $18,182,184,185,197$

targeted 208

poverty 37, 38, 66, 119, 148, 206

pregnancy 9, 38, 105

presumed consent 16, Chapter 7 passim, 251

primary care $12,126,129,130,168$, 192

privatisation $2,13,37,163,184,192$, 199, 229, 255

problematisations $11,14,184,206$, 222, 249, 250, 253, 254, 255

programmes of government 121, 206, 207,212

psychiatry $3,16,117,121-123$, 130-131, 252

psychotropic medication 122, 123

public health

campaigns 48, 50, 83, 127

discourses 98, 99110

Foucauldian approaches to $6,8,9$ policy $15,17,97,98,164,177$

strategies 14, 17, 25, 55, 118, 254

system in Ireland 13, 30, 130, 190, 196, 198, 212

public hygiene 26, 30, 33, 40

quality

of healthcare services $165,166,167$, 170, 184, 186, 187, 190, 193, 195, 196, 199, 230, 234, 235, 239,241

of life 35, 144, 227

Quality and Fairness 17, 164, 167, $170,173,176$ 
rationalities $2,7,8,16,17,18,117$, $121,124,140,144,148,164$, 173, 184, 189, 204, 207, 206, $222,249,252,254$

rationality of government $6,7,97$, 98

recovery (in mental health) 118, 123, 129

regimes

of practices 119, 121, 134

of truth 119,173

see also Dean, Mitchell

Reilly, James 36, 65, 183

resistance $2,14,17,19,72,82,91$, 92, 111, 131, 176, 239, 248, 249 see also counter-conducts responsibilisation $64,66,166,254$ risk

biomedicine and 73, 82

citizens at 1-2, 10, 48, 52, 61, 87, 99

equalisation 174

factors $63,73,83,161,162,175$

governmental strategies of 9-10, $11,15,64,65,66,77,141$, 171,208

management of $18,99,169,170$, 171, 177, 184, 191, 193, 196, 210

Rose, Nikolas 4, 6, 18, 34, 35, 74, 80, 98, 161, 183, 189, 206 see also Miller, Peter

Rousseau, Jean Jacques 14, 25-29, 31, $32,33,34,38,41$

Safefood 59, 64, 65, 84, 86, 87, 90, 91, 92

self-help 174

self-surveillance $65,66,219$

Shaping a Healthier Future 17, 164, 165, 167, 169, 172, 176

smoking 14, 16, 38, Chapter 5 passim

ban Chapter 5 passim

passive 101, 103, 104, 105

social difference 248, 253, 259

sociology of health and illness 8, 11

solidarity 18, 154, 169, 171, 172, 185, 188, 193, 194, 197-199 somatic individuality $74,75,89$

sovereign power $5,28,29,75-77$, 176

special educational needs 208, 212

statistics 5, 31, 65, 103, 204, 205, 212, 215, 218, 219-222

passim, 228

see also classification

stigmatisation 61, 62, 154

Stop the Spread 15, Chapter 4 passim

STS see Stop the Spread

subjectification $75,78,80,88,89,90$, $98,139,140,157$

subjectivities 3-10 passim, 17, 48, 90, 91, 117, 121, 131, 139, 172, 176, 205, 212, 215, 221, 227, 229, 247

subsidiarity 4, 12, 217

suicide prevention 16, 123-124, 126-127, 135

surveillance 9, 35, 38, 39, 40, 49, 55, 66, 76, 87, 97, 168, 176, 208, 215,221

taxation 182, 185, 186, 195

tax breaks 18, 185

taxpayer 232, 237

technologies of government 2, 3, 7, 10, 110, 139, 184, 189, 163, 206, 207-208, 222

of citizenship 231, 233

of domination 97

and performance 207-208

of power 140, 97

of the self $7,97,99,173,175,227$, 254

of subjectification 137, 139

Together for Health 162

two-tier health system 2, 13, 18, 182, 187, 189, 197, 199, 212, 254

UHI see Universal Health Insurance

UHI-C see Universal Health Insurance by Competition

UN Convention of the Rights of the Child 35

Universal Health Insurance 182, 183, 188, 198 
Universal Health Insurance by

Competition 182, Chapter 9 passim

Varadkar, Leo 183

VHI see Voluntary Health Insurance

Vintners Federation of Ireland 106

Voluntary Health Insurance 182, 185

welfare state $6,170,177,194$

dismantling of 141,237

and Ireland 12 wellbeing $38,56,120,123,125,171$, 172, 175,178

WHO see World Health Organization

Whole Child approach 38, 39, 41

whole system approach 36, 38

World Health Organisation 47, 60, 83, 108, 162, 173, 178 